\title{
Active Costorage of Cryogenic Propellants for Exploration
}

\author{
Ed Canavan, Rob Boyle, Shuvo Mustafi \\ Cryogenics and Fluids Branch, NASA Goddard Space Flight Center, Greenbelt, MD 20771, USA \\ Phone: 301-286-2658, Fax: 301-286-0389, e-mail: Edgar.R.Canavan@nasa.gov
}

\begin{abstract}
Long-term storage of cryogenic propellants is a critical requirement for NASA's effort to return to the moon. Liquid hydrogen and liquid oxygen provide the highest specific impulse of any practical chemical propulsion system, and thus provides the greatest payload mass per unit of launch mass. Future manned missions will require vehicles with the flexibility to remain in orbit for months, necessitating long-term storage of these cryogenic liquids. For decades cryogenic scientific satellites have used cryogens to cool instruments. In many cases, the lifetime of the primary cryogen tank has been extended by intercepting much of the heat incident on the tank at an intermediatetemperature shield cooled either by a second cryogen tank or a mechanical cryocooler. For an $\mathrm{LH}_{2} / \mathrm{LO}_{2}$ propellant system, a combination of these ideas can be used, in which the shield around the $\mathrm{LO}_{2}$ tank is attached to, and at the same temperature as, the $\mathrm{LO}_{2}$ tank, but is actively cooled so as to remove all heat impinging on the tank and shield. This configuration eliminates liquid oxygen boil-off and cuts the liquid hydrogen boil-off to a small fraction of the unshielded rate. This paper studies the concept of active costorage as a means of long-term cryogenic propellant storage. The paper describes the design impact of an active costorage system for the Crew Exploration Vehicle (CEV). This paper also compares the spacecraft level impact of the active costorage concept with a passive storage option in relation to two different scales of spacecraft that will be used for the lunar exploration effort, the CEV and the Earth Departure Stage (EDS). Spacecraft level studies are performed to investigate the impact of scaling of the costorage technologies for the different components of the Lunar Architecture and for different mission durations.
\end{abstract}

\section{INTRODUCTION}

Long-term storage of cryogenic propellants is a critical requirement for NASA's effort to return to the moon. The current Lunar Architecture consists of a Crew Exploration Vehicle (CEV) that is significantly larger than the Apollo capsule and a Lunar Lander (LL) with double the crew capacity of Apollo's LEM. In spite of this, the main launch vehicle is significantly smaller the Saturn V. The plan achieves this enhanced capacity partially by using a second launch vehicle to lift the CEV and its Service Module (SM) into orbit. However, this immediately imposes a requirement for long term storage, because, in the event of a delay in the launch of the CEV, the Earth Departure Stage (EDS), which contains 70 tons of liquid hydrogen $\left(\mathrm{LH}_{2}\right)$ and liquid oxygen $\left(\mathrm{LO}_{2}\right)$, must be able to loiter in orbit. If the propellant level drops below the required amount before the CEV launch, the mission will be lost. Additional enhancement of payload capacity is achieved by using $\mathrm{LH}_{2} / \mathrm{LO}_{2}$ rather than hypergolic propellants in the Lunar Lander descent stage, because $\mathrm{LH}_{2} / \mathrm{LO}_{2}$ has the highest specific impulse of any practical chemical rocket propellant ( $>450 \mathrm{~s}$ ), much higher than that of the $\mathrm{N}_{2} \mathrm{O}_{4} /$ Aerozine 50 (311 s) used on the LEM descent stage. Since the Lander must loiter in orbit with the EDS, it too requires long-term storage capability. Further advantage could be gained by using $\mathrm{LH}_{2} / \mathrm{LO}_{2}$ in the Lunar Lander ascent stage and the CEV-SM. Since the Lander must loiter in orbit with the EDS, it too requires long-term storage capability. Further advantage could be gained by using $\mathrm{LH}_{2} / \mathrm{LO}_{2}$ in the Lunar Lander ascent stage and the CEV-SM. Outpost missions to the moon will remain on the surface for 180 days or longer, requiring the CEV-SM and the LL ascent stage to have even longer storage times. Thus, the long term storage and handling of cryogenic propellants is a common goal across all components of the Lunar Architecture, and development of this technology is essential for the success of the lunar program.

Although cryopropellants are not new, there are still significant open questions to be resolved before they can be used on long duration missions. $\mathrm{LH}_{2} / \mathrm{LO}_{2}$ has been used as a propellant for nearly half a century, but it has been used exclusively in launch vehicles or orbit insertion stages where hold times are on the order of hours. (Hold time is the maximum time the stage can wait and still have sufficient fuel to carry out the mission.) Although $\mathrm{LH}_{2} / \mathrm{LO}_{2}$ 
has not been stored in orbit for long periods, liquid helium tanks have flown in numerous scientific missions, and have hold times of months to years, despite major disadvantages relative to large hydrogen tanks: The heat of vaporization of helium is more than an order of magnitude below that of hydrogen, and helium tanks for scientific missions are typically much smaller than propellant tanks, and thus have much larger surface-to-volume ratios. Liquid helium dewars have an external vacuum jacket that eliminate convection and gas conduction during the ground hold and launch phases. Such a shell would be mass-prohibitive for cryopropellant tanks. Instead, foam is needed for insulation within the atmosphere.

For operation in orbit, cryopropellant tanks can use many of the design features of liquid helium dewars. Most importantly, many layers of multi-layer insulation (MLI) are used to minimize the radiative heat load into the tanks. Low thermal conductivity thermal supports are used to minimize the conductive heat load onto the tanks. In a number of flight systems, two cryogens are used, with a higher temperature material absorbing the heat impinging on an intermediate shield, and reducing the heat load on the liquid helium. We term this configuration costorage. Since the heat of vaporization of higher boiling cryogens is almost always greater than that of lower boiling ones, the overall system mass can be reduced this way. Some dewars use shields "actively" cooled with mechanical cryocoolers. Although it is possible to cool to liquid helium temperatures with a mechanical cooler, the Carnot Law demands that the efficiency (watts of cooling per watt of power required) drops linearly with temperature, and in practical flight cryocoolers other losses cause efficiency to drop as $T^{2}$. Thus, there is a thermodynamic advantage to absorbing the heat at a higher temperature.

This paper presents an active costorage system. In this concept, an actively cooled shield surrounding the $\mathrm{LH}_{2}$ tank is mounted directly to the $\mathrm{LO}_{2}$ tank, and the operating temperature of the cooler is chosen to be slightly below the $\mathrm{LO}_{2}$ boiling point. Thus, the heat load on the $\mathrm{LH}_{2}$ tank is greatly reduced and the $\mathrm{LO} 2$ boiloff is eliminated. LeBar and $\mathrm{Cady}^{1}$ recently proposed an alternate co-storage concept in which the boiloff from an unshielded $\mathrm{LH}_{2} \operatorname{tank}$ is used to absorb the heat impinging on the $\mathrm{LO}_{2}$ tank, and in this way eliminate its boiloff. This idea may seem counterintuitive from a thermodynamic standpoint, because one is effectively absorbing heat at a lower temperature to cool a surface at a higher one. However, in a passive system, one is relying on cooling from cryogen that were liquefied on the ground, so thermodynamic efficiency is irrelevant; what matters is mass efficiency, and on a per mass basis, the heat of vaporization of hydrogen is more than twice that of oxygen, so it better to sacrifice $\mathrm{LH}_{2}$ to spare $\mathrm{LO}_{2}$. Depending on the size of the propellant tanks and the mission duration there is a design point where it becomes more mass-efficient to use active method over the passive method. This paper explores this trade in greater detail.

\section{SCALING OF FREESTANDING THERMAL SHIELDS}

A thermal shield reduces the radiant heat load on a tank and lowers the boil-off, at the price of some additional mass. The alternative is to allow the higher boil-off rate, and make the tank large enough to account for the lost propellant at the end of the maximum hold time. The required additional mass of propellant plus tank will grow as the maximum hold time increases, but the mass of a thermal shield is constant. Thus, there is a time, $t_{c r}$, beyond which the system with the thermal shield will have a lower overall mass. It is possible to derive a simple expression for $t_{c r}$. Without a shield, a tank that must have a certain mass of fuel at the end of time $t$ must have an additional mass

$$
\Delta m_{n s}=\frac{S_{n s} \Phi_{n s} t}{\Lambda}+\rho_{t} d_{t}\left(S_{n s}-S_{0}\right)
$$

where $S$ is the surface area of the tank, $\Phi$ is the heat flux on the tank wall, $\Lambda$ is the heat of vaporization of the cryogen, $\rho_{t}$ is the density of the tank wall, and $d_{t}$ is the thickness of the tank wall. The first term is the mass of propellant that boils off in time $t$, and the second is the additional mass of tank wall needed to accommodate this extra propellant. The subscript $n s$ refers to a tank with no shield, and the subscript 0 refers to a tank sized for zero boil-off. With a shield, the additional mass is

$$
\Delta m_{s h}=\frac{S_{s h} \Phi_{s h} t}{\Lambda}+\rho_{t} d_{t}\left(S_{s h}-S_{0}\right)+\rho_{t} d_{s} S_{s h}
$$

where $d_{s}$ is the effective thickness of the shield. The subscript $s h$ refers to a tank with a shield. The third term is the mass of the shield. (The effective thickness is the mass divided by the area times the density.) When $\Delta m_{n s}>\Delta m_{s h}$, 
the configuration with the shield has a mass advantage. We can find $t_{c r}$ by setting $\Delta m_{n s}=\Delta m_{s h}$ and solving for $t$. This gives

$$
t_{c r}=\Lambda \rho_{t} \frac{d_{t}\left(\frac{d_{s}}{d_{t}}-\frac{S_{n s}-S_{s h}}{S_{s h}}\right)}{\Phi_{n s}\left(\frac{\Phi_{n s}-\Phi_{s h}}{\Phi_{n s}}+\frac{S_{n s}-S_{s h}}{S_{s h}}\right)} .
$$

The tank surface area, $S_{s h}$ and $S_{n s}$, can be obtained from the tank volume. If the tank size is scaled so that the shape is fixed, $S_{s h}=\gamma V_{s h}^{2 / 3}$, where $\gamma$ is a geometric constant. Then

$$
S_{s h}=\gamma\left(\frac{m_{0}+\frac{S_{s h} \Phi_{s h} t}{\Lambda}}{\rho_{H_{2}}}\right)^{2 / 3},
$$

where $m_{0}$ is the mass of propellant required with no boil-off, and the second term in the numerator is the mass loss due to boil-off. This cubic equation in $S_{s h}$ has a closed-form, but very lengthy solution. Solving the equivalent equation for $S_{n s}$, and substituting reasonable values for flux with and without a shield, the quantity $\frac{S_{n s}-S_{s h}}{S_{s h}}$ is at most a few percent, even for long times $(t \sim 1 \mathrm{yr})$ and small masses $\left(m_{0} \sim 0.3\right.$ ton), where the reduction in heat flux should make the most difference. Thus, the first term in both the numerator and the denominator of the equation for $t_{c r}$ is much larger. Consequently,

$$
t_{c r} \approx \Lambda \rho_{t} \frac{d_{s}}{\Phi_{n s}-\Phi_{s h}} .
$$

Dropping this term is equivalent to ignoring the increase in the tank wall mass. Note that since the term is subtracted in the numerator and added in the denominator, this approximation overestimates the crossover time

Using somewhat conservative values for the heat flux on a cold, heavily blanketed surface from a $300 \mathrm{~K}$ background $\left(\Phi_{n s}\right)$ of $0.25 \mathrm{~W} / \mathrm{m}^{2}$, and from a background of $100 \mathrm{~K}\left(\Phi_{s h}\right)$ of $0.05 \mathrm{~W} / \mathrm{m}^{2}$, and assuming the shield is aluminum, gives $t_{c r}=d_{s} 65$ days $/ \mathrm{mm}$, where $d_{s}$ is in $\mathrm{mm}$. Thus, a system with a $1 \mathrm{~mm}$ thick aluminum shield will have a mass advantage over the same system without a shield if the required hold time is greater than 65 days. Since the thickness $d_{s}$ will scale up with mass, a shield is only advantageous for a longer time on larger tanks. To make this more quantitative, we will examine how the thickness must scale with tank size for a freestanding shield.

We considered a cylindrical shell with one end closed off by an elliptical cap and the other simplify supported. Because the structure is light, stress levels, even at a quasi-static acceleration of $15 \mathrm{~g}$ 's, are well below the yield stress for aluminum. The thickness is set by the requirement that the lowest mode frequency be kept well above the region where most of the engine-driven vibration appears. Finite element analysis indicated that the cap stiffens the end well enough that the lowest modes are close to those of a cylinder simply supported on both ends. This problem can be handled analytically. Ventsel and Krauthammer ${ }^{2}$ show that the fundamental frequency of mode $m$ is

$$
f=\frac{1}{2 \pi} \sqrt{\frac{E}{\left(1-v^{2}\right) \rho}} \frac{\sqrt{\Omega_{m}}}{R} .
$$

The quantity under the first root is purely a function of material properties: $E$ is the Young's modulus of the material, $v$ is its Poisson ratio, and $\rho$ is its density. $R$ is the radius of the cylinder and $\Omega_{m}$ is the minimum eigenvalue of the following matrix:

$$
\left(\begin{array}{ccc}
-\lambda^{2}-\frac{1-v}{2} m^{2} & \frac{1+v}{2} m \lambda & -v \lambda \\
\frac{1+v}{2} m \lambda & -m^{2}-\frac{1-v}{2} \lambda^{2} & m \\
-v \lambda & m & -1-a^{2}\left(\lambda^{2}+m^{2}\right)^{2}
\end{array}\right)
$$



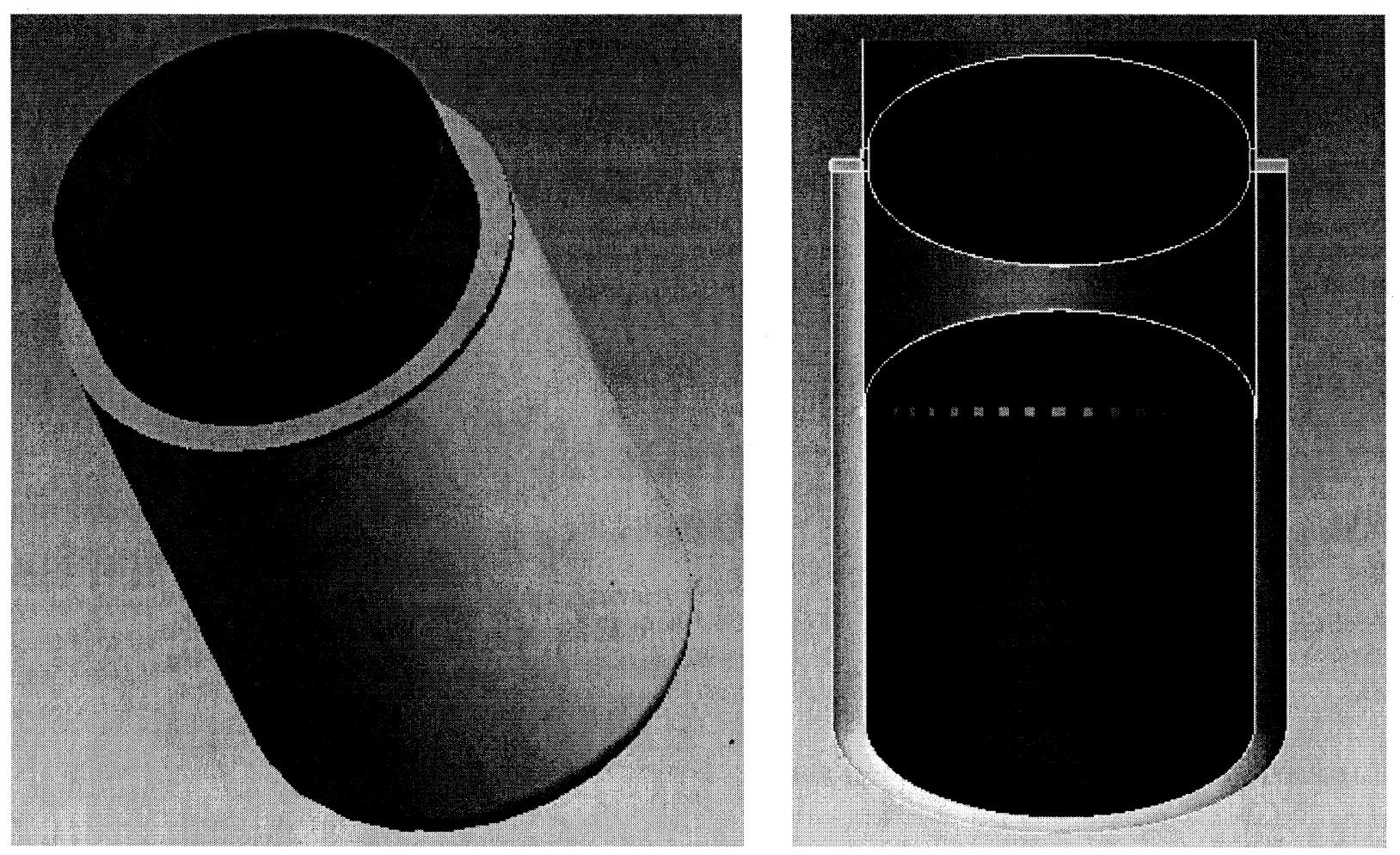

Figure 1. An active costorage unit for the CEV.

where $\lambda=\pi R / L$ and $a=\frac{d_{s}}{\sqrt{12} R}$. L is the length of the cylinder and $d_{s}$ is its thickness. An unusual feature of this solution is that the minimum frequency mode is not the one of the lowest mode number except for very long cylinders $(\lambda<<1)$. Note that if the aspect ratios $\lambda$ and $a$ are fixed, $\Omega_{m}$ is independent of scale. In this case, the minimum resonance frequency scales inversely with $R$. Using a numerical solving routine, it is straightforward to create a function that finds the thickness $d_{s}$ necessary to give a specified minimum resonance frequency. From this, we determined that as $R$ increases (with constant aspect ratio $\lambda$ ), $d_{s}$ must increase approximately as $R^{3}$ to maintain a constant resonance frequency. Thus, for a monocoque shell, mass grows as $R^{5}$ and the crossover time grows as $R^{3}$. Of course, a better design choice would be a semi-monocoque structure, in which ribs supply flexural stiffness. Such a structure has a lower areal density. Unfortunately, it is not as easily amenable to analytic techniques. Kittel, et al recently suggested a still lighter solution, in which the shield is completely flexible, and is supported between layers of MLI. Because of the shield is a thin foil, lateral conductance is limited, and heat must be collected by a periodic array of small tubes through which helium gas is circulated. Analysis of this structure is still more difficult as it does not appear to be solvable by standard finite element analysis techniques.

\section{ACTIVE COSTORAGE - PARTICULAR CONFIGURATIONS}

We now consider specific designs that make use of actively cooled shields. Figure 1 shows the concept for an active costorage unit for the CEV. Multiple units can be used depending on delta-V and packaging requirements. In this concept, the upper ellipsoidal tank is the $\mathrm{LO}_{2}$ tank and the lower cylindrical tank with ellipsoidal caps is the $\mathrm{LH}_{2}$ tank. A low thermal conductivity fiberglass skirt is used to support and isolate the $\mathrm{LO}_{2}$ tank from an external environment that is assumed to be at $300 \mathrm{~K}$. This fiberglass support also is in the load path to carry the $101 \mathrm{~K}$ thermal shield, and another low thermal conductivity fiberglass skirt that supports and isolates the $24 \mathrm{~K} \mathrm{LH}_{2} \operatorname{tank}$ from the $101 \mathrm{~K} \mathrm{LO}_{2}$ stage. The $101 \mathrm{~K}$ thermal shield and the $\mathrm{LO}_{2}$ tank are protected from the conductive and radiative load with foam and 37 layers of MLI. Mechanical cryocoolers, similar in design to currently available flight units, are used to eliminate the remaining thermal load on the $\mathrm{LO}_{2}$ tank and the thermal shield, thereby eliminating any $\mathrm{LO}_{2}$ boil-off and also providing a $101 \mathrm{~K}$ environment for the $\mathrm{LH}_{2} \operatorname{tank}$. The $\mathrm{LH}_{2}$ tank is also insulated using foam and MLI. This insulation, along with the $101 \mathrm{~K}$ environment external to the $\mathrm{LH}_{2}$ tank, enables 


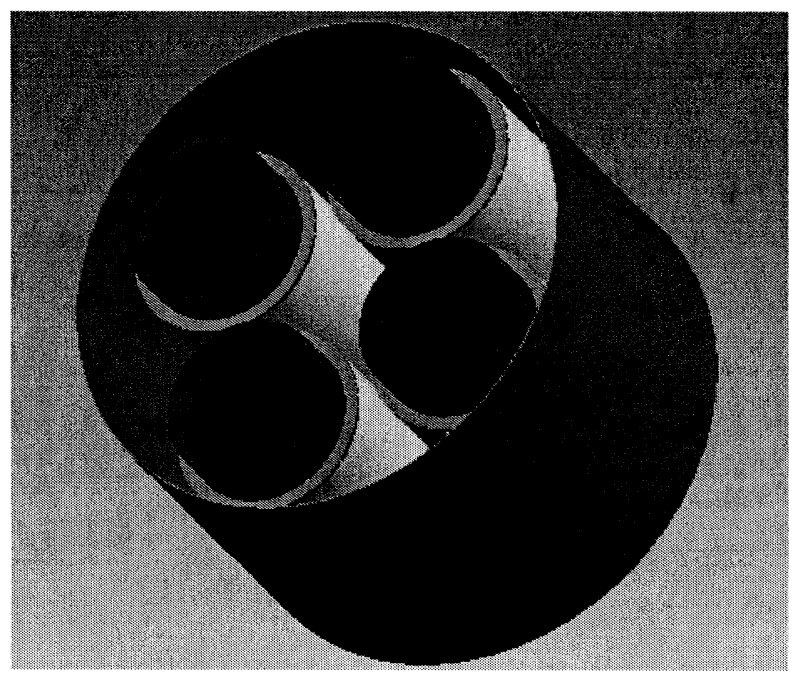

Figure 2. A 4 unit active costorage configuration in a lunar CEV-SM

an extremely low boiloff rate. For a CEV configuration that consists of four of these active costorage units, the computed thermal load on each $\mathrm{LH}_{2}$ tank is $1.1 \mathrm{~W}$. This results in a $\mathrm{LH}_{2}$ boil-off rate of $2.7 \times 10^{-6} \mathrm{~kg} / \mathrm{s}$, or about 50 $\mathrm{kg} / \mathrm{unit}(<15 \%)$ boil-off for a hold time of 183 days. Using the vented hydrogen gas to absorb some of the heat conducted along the support structure can further reduce this number ${ }^{3}$. The computed thermal load on each $\mathrm{LO}_{2}$ tank and the thermal shield is $23 \mathrm{~W}$.

This thermal load is well within the capacity of space flight cryocooler technology. For example, a relatively small $(4 \mathrm{~kg})$ cooler with significant flight heritage has approximately half this capacity at $101 \mathrm{~K}$. These units have a specific power of approximately $12 \mathrm{~W} / \mathrm{W}$ at $100 \mathrm{~K}^{4}$, and thus two of them would require $276 \mathrm{~W}$ of input power to absorb $23 \mathrm{~W}$. Coolers of similar design, but with a compressor of more than five times the capacity ${ }^{5}$ have been demonstrated up to TRL 6. Flight cryocoolers have operated on orbit for longer than five years, and have had only one failure to date. They are used here to extend the useful service life of the propellant tanks, but are not a safety critical item. In a simple system where each cooler is directly linked to one unit, a cryocooler failure would require early crew return, but would not cause a safety hazard. Cross strapping the coolers so that each one could cool the other three units would increase complexity of integration, but would easily make the system two-fault tolerant. There is however a mass penalty for using this configuration over the passive storage option primarily because of the thermal shield mass. This mass penalty has to be compared to the mass penalty of allowing a higher $\mathrm{LH}_{2}$ boiloff as is explained in the previous section. This configuration was also used to model usage for the much larger scale of the EDS, with one large costorage unit.

Figure 2 show the packaging of a 4 unit active costorage configuration in a lunar CEV-Service Module (SM). This configuration allows a central thrust tube. The CEV-SM dimension in this configuration is the closest to the dimensions for the CEV-SM that are presented in Exploration System Architecture Study (ESAS) report, $5.5 \mathrm{~m}$ in diameter and $3.46 \mathrm{~m}$ in length. The CEV-SM for the 4 unit configuration has a $5.5 \mathrm{~m}$ diameter with a length of 3.98 $\mathrm{m}$. Figure 3 shows the variation of CEV wet launch mass with the number of units. Between 3 and 8 units, the 4 unit configuration provides the optimized minimum launch mass of about 23.4 metric tons. The one and two unit configurations can have considerably lower launch masses of about 21.6 and 22.5 metric tons respectively. However, the corresponding CEV-SMs while having the same diameter of $5.5 \mathrm{~m}$ will have a length of $7.34 \mathrm{~m}$ and $4.59 \mathrm{~m}$ respectively. The analysis leading to figure 3 involves a spreadsheet design program that iteratively obtains the active co-storage design based on structural and thermal requirements along with the design constraints such as delta $-\mathrm{V}$ requirements and fairing diameters as is specified in the ESAS report and the CEV - Smart Buyer program.

\section{CEV AND EDS LAUNCH MASS - DEPENDENCE ON HOLD TIME}

The requirements on the hold times of each component of the lunar architecture have not yet been defined. The goal for stays on the lunar surface is at least 180 days. The requirement for the maximum time between the launch of the 
EDS (with the Lander) and the CEV is in flux, but periods as long as 95 days have been discussed. Obviously, a longer period is desirable, as it affords much greater flexibility. With a 95 day hold time requirement on the EDS, the Lander descent stage would require 98 days (including the transit to the moon), and a cryogenic ascent stage and CEV would require nearly 280 days. For the CEV and Lander ascent stages, developing a technically feasible design that can achieve these long hold times while still providing a significant mass advantage is necessary for the cryopropellant option.

The spreadsheet program that was used to generate figure 3 was also used to perform a parametric study on the variation of total launch CEV and EDS wet masses with mission duration for both active costorage and passive storage. The wet launch mass for active costorage includes the mass associated with the $101 \mathrm{~K}$ thermal shield and the associated cryocooler components. The wet launch mass for passive costorage does not include the $101 \mathrm{~K}$ themal shield and the cryocooler components but instead it includes the mass of the extra propellant and the increased mass of tank volume that is required to account for the extra $\mathrm{LH}_{2}$ boil-off that results from the lack of the thermal shield. The corresponding difference in mass between the active costorage and the passive storage concepts can be traced back to an overall difference in the spacecraft wet launch mass between these storage configurations for the CEV and the EDS. The required wet launch mass of the spacecraft changes with the mission duration. Figures 4 and 5 show the variation of the wet launch mass of a CEV sized vehicle and an EDS sized vehicle with mission duration respectively. Figure 4 indicates that at about 210 days it is more mass advantageous to use the 4 unit active costorage configuration than a 4 unit passive storage configuration. It is interesting to note that $730 \mathrm{Kg}$ of additional active costorage and propellant mass out of a CEV wet launch mass of 23 tons can extend the mission duration from 180 to 365 days. Figure 5 shows at about 355 days it is more mass advantageous to use active costorage than passive storage. An additional 11.7 tons of additional active costorage and propellant mass out of an EDS wet launch mass of 167 tons can extend the mission duration from 95 days to 730 days.

\section{CEV Wet Launch Mass [Kg]}

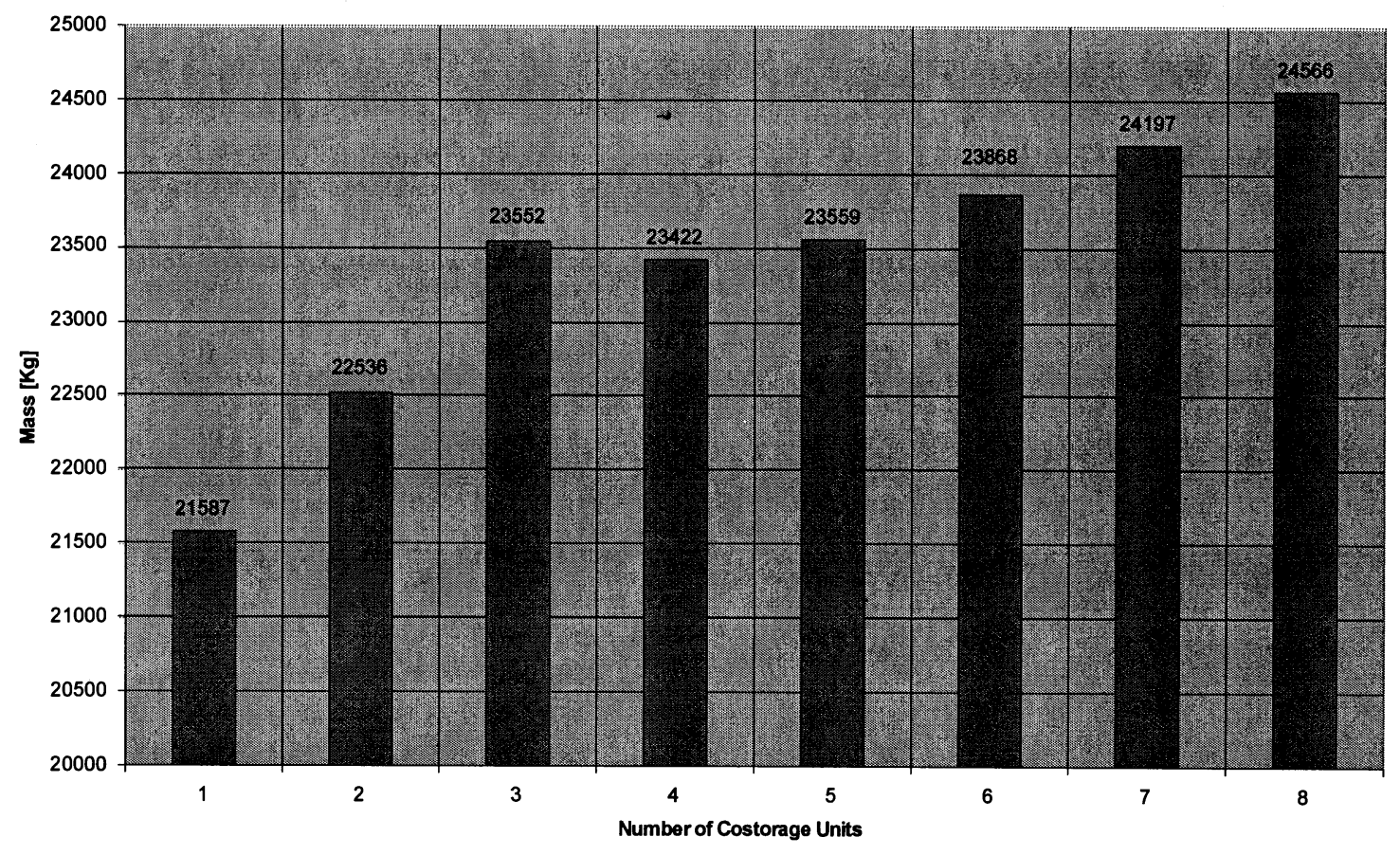

Figure 3. CEV wet launch mass variation with number of active costorage units 
CEV Launch Mass required for TEI Vs. Duration on the Moon

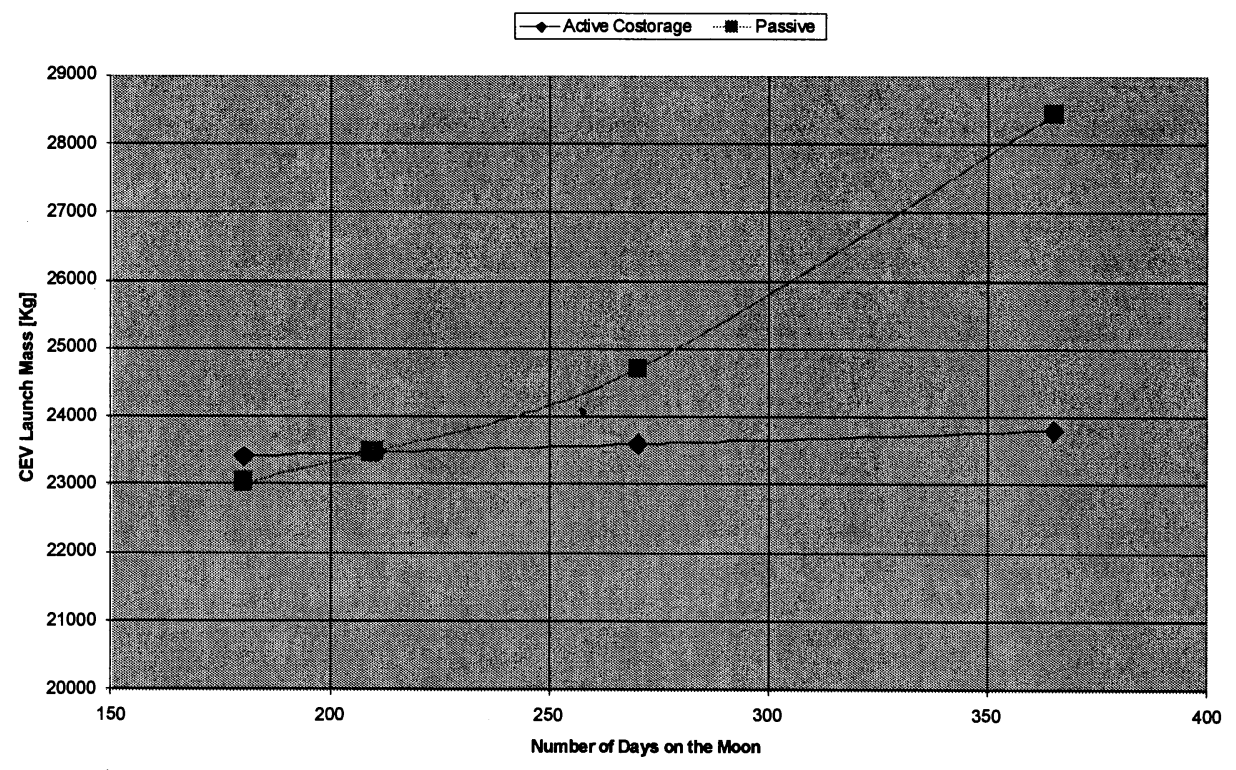

Figure 5. Variation of CEV launch mass with mission duration for the active costorage and passive storage options.

\section{CONCLUSIONS}

In active co-storage, all heat leaks into a $\mathrm{LH}_{2}$ tank are intercepted at the $\mathrm{LO}_{2}$ tank, and a thermal shield connected to EDS Launch Mass required for LEO circularization and TU of 58 tons Vs Loiter Duration

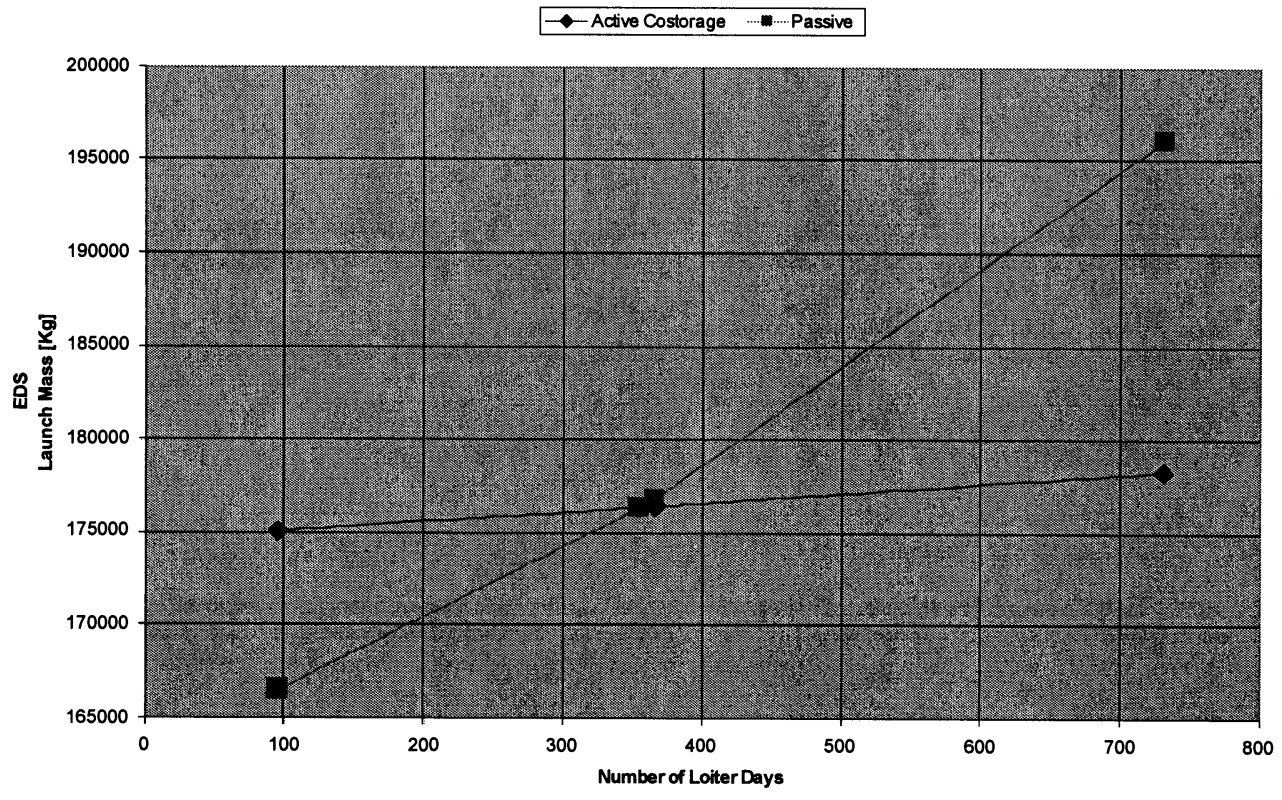

Figure 4. Variation of EDS launch mass with mission duration for the active costorage and passive storage options. 
it. Heat loads into the $\mathrm{LO}_{2}$ tank are absorbed by mechanical cryocoolers at a temperature slightly below the boiling point of the $\mathrm{LO}_{2}$, so that oxygen boil-off is completely eliminated. Hydrogen boil-off is reduced to the point where the mass loss over periods on the order of a year is tolerable. Absorbing heat at $100 \mathrm{~K}$ only keeps the cryocooler power requirements modest and can be accomplished with current technology. Existing cryocooler designs need only be adjusted to optimize them for operation at $100 \mathrm{~K}$. Given the high reliability of spaceflight cryocoolers, the ability to create two-fault tolerant systems with multiple coolers, and the non-critical nature of a cooler failure, the risk of actively cooled systems appears low. The primary alternative to an actively cooled system is a passive system in which the hydrogen boil-off gas is used to absorb heat impinging on the $\mathrm{LO}_{2}$ tank. This system has a mass advantage for short hold times because it does not include a shield, but active costorage is less massive for long hold times. Analysis shows that the crossover time is proportional to the areal density of the shield. A simple monocoque shell is fairly lightweight for small tanks, but its thickness must grow rapidly with scale to maintain a minimum resonance frequency. Other, lighter weight structures are available that maintain a low areal density at larger scales. Parametric studies have been performed of an active costorage design at CEV and EDS scales. These much more detailed models conform the predicted trends, such as a much longer cross over time at larger scales.

\section{REFERENCES}

$\left.{ }^{1}\right)$ Lebar, J. F. and Cady, E. C., "The advanced cryogenic evolved stage (ACES) - a low-cost, low-risk approach to space exploration launch", (submitted to Cryogenics).

${ }^{2}$ Ventsel, E. and Krauthammer, T., Thin Plates and Shells - Theory, Analysis, and Applications, Marcel Dekker, Inc., New York, 2001.

${ }^{3}$ Canavan, E. R. and Miller, F. K., "Optimized heat interception for cryogen tank support", presented at the Cryogenics Engineering Conference, Chattanooga, TN 2007.

${ }^{4}$ Salerno, L. J., Kittel, P., Helvensteijn, B. P. M., and Kashani, A., "Performance testing of a lightweight, high efficiency cooler", Proceedings of the $12^{\text {th }}$ International Cryocooler Conference, Cambridge, MA June 18-20, 2002.

${ }^{5}$ Jaco, C., et al. "High capacity staged pulse tube" Advances in Cryogenic Engineering 49 pp. 1263-8 (2004). 